\title{
CURVATURE PROPERTIES OF ALMOST RICCI-LIKE SOLITONS WITH TORSE-FORMING VERTICAL POTENTIAL ON ALMOST CONTACT B-METRIC MANIFOLDS
}

\author{
MANCHO MANEV
}

\begin{abstract}
Aвstract. A generalization of $\eta$-Ricci solitons is considered involving an additional metric and functions as soliton coefficients. The soliton potential is torseforming and orthogonal to the contact distribution of the almost contact B-metric manifold. Then such a manifold can also be considered as an almost Einstein-like manifold, a generalization of an $\eta$-Einstein manifold with respect to both B-metrics and functions as coefficients. Necessary and sufficient conditions are found for a number of properties of the curvature tensor and its Ricci tensor of the studied manifolds. Finally, an explicit example of an arbitrary dimension is given and some of the results are illustrated.
\end{abstract}

\section{INTRODUCTION}

A notion of a Ricci soliton is a natural extension of an Einstein metric on a smooth manifold. Recently, Hamilton's concept of the Ricci flow introduced on Riemannian manifolds in [14], has been developed by many authors for (pseudo)Riemannian manifolds with various additional tensor structures (e.g. [1], [2], [3], [4], [5], [6], [16], [25], [26], [27], [28], [29]).

A significant part of these works are for $\eta$-Ricci solitons on manifolds with a horizontal distribution $\operatorname{ker} \eta$ for some 1-form $\eta$. Moreover, the manifold is of Kenmotsu type, where the soliton potentials can be torse-forming and orthogonal to ker $\eta$. Torse-forming vector fields are defined by a certain recurrent condition for their covariant derivative with respect to the Levi-Civita connection of the basic metric [30]. This research has been expanded to study various properties of the curvature tensor and its Ricci tensor compatible with the additional tensor structures.

In [18], the author begins the study of Ricci-like solitons on almost contact Bmetric manifolds, whose Reeb vector field $\xi$ is the soliton potential. These solitons are a generalization of $\eta$-Ricci solitons because use also the associated B-metric of the manifold. In [20], the author generalizes the Ricci-like solitons to almost Ricci-like solitons on the considered manifolds, considering the soliton coefficients as functions. Then, he studies the case when the potential is torse-forming and vertical, i.e. collinear to $\xi$.

Following [18], the only basic class of almost contact B-metric manifolds that allows a torse-forming Reeb vector field is $\mathcal{F}_{5}$, denoted according to the GanchevMihova-Gribachev classification [11]. This class is considered in the present work and $\mathcal{F}_{5}$ is the counterpart of the class of $\beta$-Kenmotsu manifolds among the classes of almost contact metric manifolds. Ricci solitons and $\eta$-Ricci solitons on manifolds with almost contact or almost paracontact structures, both of Kenmotsu type, have been studied in a number of works (e.g. [1], [2], [5], [15], [25], [27]).

In the present paper, our aim is to study curvature properties of almost Riccilike solitons whose potential is torse-forming and vertical on almost contact Bmetric manifolds. The paper is organized as follows. In Section 2, we recall basic definitions and facts for the considered manifolds and the tensor structures on 
them. In Section 3, we investigate a series of properties of the curvature tensor and its Ricci tensor on the manifolds under study. In Section 4, we give an explicit example of a smooth manifold of the considered type and comment on the results for this example supporting the relevant assertions in the previous section.

\section{Preliminaries}

2.1. Torse-forming vertical vector fields on almost contact B-metric manifolds. We consider almost contact B-metric manifolds denoted by $(M, \varphi, \xi, \eta, g)$. This means that any $M$ is a $(2 n+1)$-dimensional smooth manifold equipped with an almost contact structure and a B-metric, i.e. a pseudo-Riemannian metric of signature $(n+1, n)$, satisfying the following conditions [11]

$$
\begin{array}{cl}
\varphi \xi=0, \quad & \varphi^{2}=-\imath+\eta \otimes \xi, \quad \eta \circ \varphi=0, \quad \eta(\xi)=1, \\
& g(\varphi x, \varphi y)=-g(x, y)+\eta(x) \eta(y),
\end{array}
$$

where $\iota$ is the identity transformation on $\Gamma(T M)$.

In the latter equality and further, $x, y, z, w$ will stand for arbitrary elements of $\Gamma(T M)$ or vectors in the tangent space $T_{p} M$ of $M$ at an arbitrary point $p$ in $M$.

An important characteristic of these manifolds is the existence of an associated B-metric $\tilde{g}$ of $g$ defined by

$$
\tilde{g}(x, y)=g(x, \varphi y)+\eta(x) \eta(y) .
$$

A classification of almost contact B-metric manifolds is given in [11]. It is made with respect to the $(0,3)$-tensor $F$ defined by $F(x, y, z)=g\left(\left(\nabla_{x} \varphi\right) y, z\right)$. Each basic class $\mathcal{F}_{i}, i \in\{1,2, \ldots, 11\}$ contains the special class $\mathcal{F}_{0}$, determined by $F=0$, and known as the class of the cosymplectic B-metric manifolds.

A vector field $\vartheta$ on a (pseudo-)Riemannian manifold $(M, g)$ is called torse-forming vector field if it satisfies the following condition for an arbitrary vector field $x$, a differentiable function $f$ and a 1 -form $\gamma$

$$
\nabla_{x} \vartheta=f x+\gamma(x) \vartheta,
$$

where $\nabla$ is the Levi-Civita connection of $g$ [30]. The 1 -form $\gamma$ is called the generating form and the function $f$ is called the conformal scalar of $\vartheta$ [22].

In [18], manifolds $(M, \varphi, \xi, \eta, g)$ with torse-forming Reeb vector field $\xi$ are studied. Then, $\gamma$ has the form $\gamma=-f \eta$. There, it is deduced that the class of the considered manifolds is $\mathcal{F}_{1} \oplus \mathcal{F}_{2} \oplus \mathcal{F}_{3} \oplus \mathcal{F}_{5} \oplus \mathcal{F}_{6} \oplus \mathcal{F}_{10}$ and only $\mathcal{F}_{5}$ can contain such manifolds among the basic classes. If $(M, \varphi, \xi, \eta, g)$ is an $\mathcal{F}_{5}$-manifold with torse-forming $\xi$, then we have

$$
\left(\nabla_{x} \varphi\right) y=-f\{g(x, \varphi y) \xi+\eta(y) \varphi x\} .
$$

In this case, it is found that the condition for Ricci-symmetry is equivalent to the Einstein condition on this Einstein-like manifold.

We further exclude from our consideration the trivial case when $f=0$, since it implies the parallelism of $\vartheta$.

In the rest part of our work, we consider a torse-forming vector field $\vartheta$, i.e. (2) is valid. Moreover, $\vartheta$ is pointwise collinear with $\xi$, i.e. $\vartheta=k \xi$, where $k$ is a nonzero pointwise differentiable function on $M$. Therefore $\vartheta$ belongs to the vertical distribution $H^{\perp}=\operatorname{span} \xi$, which is orthogonal to the contact distribution $H=\operatorname{ker} \eta$ with respect to $g$. For that reason, we call such a vector field $\vartheta$ vertical.

Theorem 2.1 ([20]). Let a vector field $\vartheta$ on $(M, \varphi, \xi, \eta, g)$ be torse-forming with a conformal scalar $f$ and generating form $\gamma$. Moreover, let $\vartheta$ be vertical, i.e. $\vartheta=k \xi$, where $k$ is a differentiable function on $M$. Then we have:

(i) $\xi$ is a geodesic vector field and $\eta$ is a closed 1-form; 
(ii) $\xi$ is a torse-forming vector field with conformal scalar $f / k$ and generating form $\gamma=-(f / k) \eta$

(iii) $k$ is constant.

(iv) the following equalities for the curvature tensor $R$, the Ricci tensor $\rho$ and the sectional curvature K are valid:

$$
\begin{aligned}
& R(x, y) \xi=-\frac{1}{k^{2}}\left\{\left[k \mathrm{~d} f(x)+f^{2} \eta(x)\right] \varphi^{2} y-\left[k \mathrm{~d} f(y)+f^{2} \eta(y)\right] \varphi^{2} x\right\} \\
& R(x, \xi) \xi=\frac{1}{k^{2}}\left\{k \mathrm{~d} f(\xi)+f^{2}\right\} \varphi^{2} x, \\
& \rho(y, \xi)=-\frac{1}{k^{2}}\left\{(2 n-1) k \mathrm{~d} f(y)+\left[k \mathrm{~d} f(\xi)+2 n f^{2}\right] \eta(y)\right\}, \\
& \rho(\xi, \xi)=-\frac{2 n}{k^{2}}\left\{k \mathrm{~d} f(\xi)+f^{2}\right\}, \\
& K(\xi, x)=-\frac{1}{k^{2}}\left\{k \mathrm{~d} f(\xi)+f^{2}\right\},
\end{aligned}
$$

i.e. the scalar curvature of any $\xi$-section $(\xi, x), x \notin H^{\perp}$, does not depend on $x$.

Remark 2.2. Let us recall [20] that a torse-forming vertical vector field $\vartheta$ on $(M, \varphi, \xi, \eta$, $g$ ) is called regular if its generating function $f$ and the proportionality constant $k$ with $\xi$ satisfy the condition $k \mathrm{~d} f(\xi)+f^{2} \neq 0$. Then, $R(x, \xi) \xi$ in (5) does not degenerate for any $x$. For example, if we have $f=(1+\sqrt{3}) k /(2 t)$ (respectively, $f=k / t)$ then $\vartheta$ with this conformal scalar $f$ is regular (respectively, it is not regular).

According to Theorem 2.1(ii) and (3) for $(M, \varphi, \xi, \eta, g) \in \mathcal{F}_{5}$, we have the following

$$
\begin{aligned}
& \left(\nabla_{x} \varphi\right) y=-\frac{f}{k}\{g(x, \varphi y) \xi+\eta(y) \varphi x\} \\
& \nabla_{x} \vartheta=-f \varphi^{2} x
\end{aligned}
$$

2.2. Einstein-like almost contact B-metric manifolds. In [18], it is said that $(M, \varphi$, $\xi, \eta, g)$ is Einstein-like if its Ricci tensor $\rho$ has the following form for some triplet of constants $(a, b, c)$ :

$$
\rho=a g+b \tilde{g}+c \eta \otimes \eta .
$$

In particular, when $b=0$ and $b=c=0$, the manifold is called an $\eta$-Einstein manifold and an Einstein manifold, respectively. If $a, b, c$ are functions on $M$, then the manifold is called almost Einstein-like, almost $\eta$-Einstein and almost Einstein, respectively.

Other particular cases of Einstein-like types of the considered manifolds are given in [21] and [17]. For example, when $a=b=0$, the manifold is called $v$ Einstein for short from vertical-Einstein since $\eta \otimes \eta$ is the vertical component of $g$ and $\tilde{g}$.

Now we introduce the notion of an almost $v$-Einstein manifold when the condition (11) is satisfied for $a=b=0$ and a function $c$ on $M$.

As a consequence of (11) we obtain that the corresponding scalar curvature and its associated quantity on an almost Einstein-like manifold have the form:

$$
\tau=(2 n+1) a+b+c, \quad \tau^{*}=-2 n b,
$$

where $\tau^{*}:=g^{i j} \rho\left(e_{i}, \varphi e_{j}\right)$ in an arbitrary basis $\left\{e_{i}\right\}, i \in\{1,2, \ldots, 2 n+1\}$ of $T_{p} M$. 
2.3. Ricci-like solitons with torse-forming vertical potential on almost contact B-metric manifolds. According to [19], an almost contact B-metric manifold ( $M$, $\varphi, \xi, \eta, g)$ is said to admit a Ricci-like soliton with potential vector field $\vartheta$ if its Ricci tensor $\rho$ satisfies the following condition for a triplet of constants $(\lambda, \mu, v)$

$$
\frac{1}{2} \mathcal{L}_{\vartheta} g+\rho+\lambda g+\mu \tilde{g}+v \eta \otimes \eta=0
$$

where $\mathcal{L}$ denotes the Lie derivative.

If $\mu=0$ (respectively, $\mu=v=0$ ), then (13) defines an $\eta$-Ricci soliton (respectively, a Ricci soliton) on $(M, \varphi, \xi, \eta, g)$.

If $\lambda, \mu, v$ are functions on $M$, then the soliton is called almost Ricci-like soliton, almost $\eta$-Ricci soliton and almost Ricci soliton, respectively [24].

A Ricci soliton is called shrinking, steady or expanding depending on whether $\lambda$ is negative, zero or positive, respectively [8].

Similar to the case of a Ricci-like soliton with potential $\vartheta=\xi$, studied in [18], we recall the following more general result for almost Ricci-like solitons with torse-forming vertical potential.

Theorem 2.3 ([20]). Let $(M, \varphi, \xi, \eta, g)$ be $(2 n+1)$-dimensional and $\vartheta$ be a vector field on $M$, which is vertical with a constant $k=\eta(\vartheta)$ as well as torse-forming with a function $f$ as a conformal scalar. Moreover, let $a, b, c, \lambda, \mu, v$ be functions on $M$ that satisfy the following equalities:

$$
\begin{aligned}
& a+\lambda+f=0, \quad b+\mu=0, \quad c+v-f=0, \\
& \lambda+\mu+v=-a-b-c=\frac{2 n}{k^{2}}\left\{k \mathrm{~d} f(\xi)+f^{2}\right\} .
\end{aligned}
$$

Then, $M$ admits an almost Ricci-like soliton with potential $\vartheta$ and functions $(\lambda, \mu, v)$ if and only if $M$ is almost Einstein-like with functions $(a, b, c)$.

Then, (11) and (14) yield the following expression of the Ricci tensor

$$
\rho(x, y)=(\lambda+f) g(\varphi x, \varphi y)-\mu g(x, \varphi y)-(\lambda+\mu+v) \eta(x) \eta(y) .
$$

Remark 2.4. By Remark 2.2 and (15) we have that $\vartheta$ is regular if and only if both sums $a+b+c$ and $\lambda+\mu+v$ vanish.

Corollary 2.5 ([20]). Under the hypothesis of Theorem 2.3 we have in particular:

(i) The manifold admits an almost $\eta$-Ricci soliton with potential $\vartheta$ if and only if it is almost $\eta$-Einstein.

(ii) The manifold admits an almost Ricci soliton with potential $\vartheta$ and function $\lambda$ if and only if it is almost $\eta$-Einstein with functions $(a, b, c)=(-\lambda-f, 0, f)$, which is not almost Einstein.

(iii) The manifold is almost Einstein with function a if and only if it admits an almost $\eta$-Ricci soliton with potential $\vartheta$ and functions $(\lambda, \mu, v)=(-a-f, 0, f)$, which is not almost Ricci soliton with potential $\vartheta$.

Corollary 2.6 ([20]). Under the hypothesis of Theorem 2.3 we have that $M$ is almost Einstein-like with functions $(a, b, c)$ and scalar curvature $\tau$. Then the conformal scalar $f$ satisfies the following equation

$$
k f^{\prime}+f^{2}=k^{2}\left(a-\frac{\tau}{2 n}\right),
$$

where $f^{\prime}$ is the derivative of the function $f=f(t)$ and $t$ is a coordinate on $H^{\perp}$ and the sectional curvature of an arbitrary $\xi$-section is

$$
K_{\xi}=\frac{\tau}{2 n}-a
$$


3. Some CURVATURE Properties of Almost RiCCI-LiKe SOlitons With TORSE-Forming VERTICAL POTENTIAL ON ALMOST CONTACT B-METRIC MANIFOLDS

Throughout this section, we assume that $(M, \varphi, \xi, \eta, g)$ is a $(2 n+1)$-dimensional manifold belonging to the class $\mathcal{F}_{5} \backslash \mathcal{F}_{0}$ and admitting an almost Ricci-like soliton with potential $\vartheta$, which is torse-forming with a non-zero conformal scalar $f$ and a vertical one with a non-zero constant coefficient $k$.

3.1. Locally Ricci symmetric manifolds. If consider the condition for locally Ricci symmetry, i.e. $\nabla \rho$ vanishes, we immediately obtain the following

Theorem 3.1. The following characteristics of the manifold $(M, \varphi, \xi, \eta, g)$ are equivalent:

(i) It is Ricci symmetric.

(ii) It admits an almost $\eta$-Ricci soliton with functions $(\lambda, \mu, v)$, where

$$
\lambda=-\frac{\tau}{2 n+1}-f, \quad \mu=0, \quad v=f
$$

and the scalar curvature $\tau$ of $M$ is the following constant

$$
\tau=-\frac{2 n(2 n+1)}{k^{2}}\left\{k \mathrm{~d} f(\xi)+f^{2}\right\} .
$$

(iii) It is Einstein with constant $a$ and $\tau=(2 n+1) a$.

Proof. Because of Theorem 2.1(ii)-(iii) and (15), we get

$$
\left(\nabla_{x} \gamma\right)(y)=+\frac{1}{k^{2}} f^{2} g(\varphi x, \varphi y)+\left[\frac{1}{k^{2}} f^{2}-\frac{1}{2 n}(\lambda+\mu+v)\right] \eta(x) \eta(y) .
$$

Using the latter equality, Theorem 2.1(ii)-(iii), (9), (15) and (16), we compute the covariant derivative of the Ricci tensor with respect to $\nabla$ of an $\mathcal{F}_{5}$-manifold admitting an almost Ricci-like soliton with torse-forming vertical potential as follows

$$
\begin{aligned}
\left(\nabla_{x} \rho\right)(y, z)= & \left\{\mathrm{d} \lambda(x)-\left[\frac{1}{k} f^{2}-\frac{k}{2 n}(\lambda+\mu+v)\right] \eta(x)\right\} g(\varphi y, \varphi z) \\
& -\mathrm{d} \mu(x) g(y, \varphi z)-\mathrm{d}(\lambda+\mu+v)(x) \eta(y) \eta(z) \\
& +\frac{f}{k}\{\mu[g(x, \varphi y) \eta(z)+g(x, \varphi z) \eta(y)] \\
& +(\mu+v-f)[g(\varphi x, \varphi y) \eta(z)+g(\varphi x, \varphi z) \eta(y)]\} .
\end{aligned}
$$

As consequences of the latter formula we have

$$
\begin{aligned}
& \left(\nabla_{x} \rho\right)(\varphi y, \varphi z)=-\left\{\mathrm{d} \lambda(x)-\left[\frac{1}{k} f^{2}-\frac{k}{2 n}(\lambda+\mu+v)\right] \eta(x)\right\} g(\varphi y, \varphi z) \\
& \quad+\mathrm{d} \mu(x) g(y, \varphi z),
\end{aligned}
$$

Therefore, taking into account (20), we establish that $\rho$ is $\nabla$-parallel (i.e. (i) holds) if and only if the soliton functions are:

$$
\lambda=-f+\text { const }, \quad \mu=0, \quad v=f \text {. }
$$

Bearing in mind (21), we deduce that $\lambda$ and $v$ (just like $f$ ) are horizontal constants, i.e. constants on $H$, and thus they are vertical functions, i.e. on $H^{\perp}$.

By (14) we obtain that the constant in (21) is $-a$ and $b=c=0$ is true. Hence, the manifold under consideration is Einstein with the constant $a$, which is equal to $\tau /(2 n+1)$ due to (12). Therefore, we obtain (19) using (15), and this completes the proof. 
3.2. Manifolds of Ricci semi-symmetric type. A manifold is called Ricci semisymmetric if the following condition is satisfied $R(x, y) \cdot \rho=0$, where $R(x, y)$ is considered as a field of linear operators, acting on the Ricci $(0,2)$-tensor $\rho$.

Let us consider the condition $R\left(\xi_{,}\right) \cdot \rho=0$, i.e. it is valid the following

$$
\rho(R(\xi, x) y, z)+\rho(y, R(\xi, x) z)=0 .
$$

Theorem 3.2. Let $(M, \varphi, \xi, \eta, g)$ be with a regular potential $\vartheta$ and scalar curvature $\tau$. Then the following characteristics of the manifold are equivalent:

(i) The condition $R(\xi,.) \cdot \rho=0$ is satisfied.

(ii) The manifold admits an almost $\eta$-Ricci soliton with functions $(\lambda, \mu, v)=(-f, 0, f-$ $\tau)$, where the scalar curvature $\tau$ is the following non-vanishing function on $H^{\perp}$

$$
\tau=-\frac{2 n}{k^{2}}\left\{k \mathrm{~d} f(\xi)+f^{2}\right\} .
$$

(iii) The manifold is almost $v$-Einstein with functions $(a, b, c)=(0,0, \tau)$, where $\tau$ is the function on $\mathrm{H}^{\perp}$ given in (22).

Proof. By using of (4) and (15), we get the following

$$
R(\xi, y) z=-\frac{1}{k^{2}}\left[k \mathrm{~d} f(\xi)+f^{2}\right]\left\{\eta(z) \varphi^{2} y-g(\varphi y, \varphi z) \xi\right\} .
$$

Then, applying (16) and (23), we obtain the following equality

$$
\begin{aligned}
& {\left[k \mathrm{~d} f(\xi)+f^{2}\right] }\{b[\eta(z) g(x, \varphi y)+\eta(y) g(x, \varphi z)] \\
&-a[\eta(z) g(\varphi x, \varphi y)+\eta(y) g(\varphi x, \varphi z)]\}=0,
\end{aligned}
$$

which for $z=\xi$ implies

$$
\left[k \mathrm{~d} f(\xi)+f^{2}\right]\{b g(x, \varphi y)-a g(\varphi x, \varphi y)\}=0 .
$$

Since $\vartheta$ is regular, the later equality is valid for arbitrary $x$ and $y$ if and only if $a=b=0$. Therefore, we have $\rho=c \eta \otimes \eta$ and $\tau=c$, i.e. the manifold is $\mathrm{v}$-Einstein. Because of (17), we get (22) and $\tau \neq 0$ due to the regularity of $\vartheta$. Then, taking into account (12), we establish that (i) is equivalent to (iii). (15).

Finally, the equivalence between (ii) and (iii) follows by virtue of (14) and

3.3. Manifolds with a cyclic parallel Ricci tensor. A non-vanishing Ricci tensor $\rho$ is called cyclic parallel if it satisfies the condition [13]

$$
\left(\nabla_{x} \rho\right)(y, z)+\left(\nabla_{y} \rho\right)(z, x)+\left(\nabla_{z} \rho\right)(x, y)=0 .
$$

It is known that (24) is equivalent to requiring that the Ricci tensor is a Killing tensor, i.e. $\left(\nabla_{x} \rho\right)(x, x)=0$.

Theorem 3.3. The following characteristics of the manifold $(M, \varphi, \xi, \eta, g)$ are equivalent:

(i) The Ricci tensor of the manifold is cyclic parallel.

(ii) The manifold admits an almost Ricci-like soliton with vertical functions $(\lambda, \mu, v)$ satisfying the following equations

$$
\begin{gathered}
\mathrm{d} \lambda=-\left\{f^{\prime}+\frac{2 f}{k}(\mu+v-f)\right\} \eta, \\
\mathrm{d} \mu=\frac{2 f}{k} \mu \eta, \quad \mathrm{d} v=\left\{f^{\prime}+\frac{2 f}{k}(v-f)\right\} \eta .
\end{gathered}
$$

(iii) The manifold is almost Einstein-like with vertical functions $(a, b, c)$ satisfying the following equations

$$
\mathrm{d} a=-\frac{2 f}{k}(b+c) \eta, \quad \mathrm{d} b=\frac{2 f}{k} b \eta, \quad \mathrm{d} c=\frac{2 f}{k} c \eta .
$$


Proof. Applying (20) to (24), we obtain that the condition $\rho$ to be cyclic parallel is equivalent to the following condition for the cyclic sum of $x, y$ and $z$

$$
\begin{aligned}
\underset{x, y, z}{ }\{ & {\left[\mathrm{d}(\lambda+f)(x)+\frac{2 f}{k}(\mu+v-f) \eta(x)\right] g(\varphi y, \varphi z) } \\
& \left.-\left[\mathrm{d} \mu(x)-\frac{2 f}{k} \mu \eta(x)\right] g(y, \varphi z)-\mathrm{d}(\lambda+\mu+v)(x) \eta(y) \eta(z)\right\}=0,
\end{aligned}
$$

which by substituting $x, y$ and $z$ consecutively with $\xi$ yields $\mathrm{d}(\lambda+\mu+v)=0$ and

$$
\mathrm{d}(\lambda+f)(\xi)=-\frac{2 f}{k}(\mu+v-f), \quad \mathrm{d} \mu(\xi)=\frac{2 f}{k} \mu .
$$

Taking into account (27), then (26) is valid for arbitrary $x, y$ and $z$ if and only if

$$
\mathrm{d}(\lambda+f)(x)=\mathrm{d}(\lambda+f)(\xi) \eta(x), \quad \mathrm{d} \mu(x)=\mathrm{d} \mu(\xi) \eta(x) .
$$

Bearing in mind (28) and (15), we obtain that the soliton functions $\lambda, \mu$ and $\nu$ satisfy (25). Therefore they are horizontal constants, i.e. they depend only on the coordinate of $H^{\perp}$ just as $f$. This means that the equivalence between (i) and (ii) holds true.

Finally, the equivalence between (ii) and (iii) follows from Theorem 2.3.

3.4. Manifolds with a Ricci tensor of Codazzi type. A non-vanishing Ricci tensor $\rho$ is called of Codazzi type if it satisfies the condition [13]

$$
\left(\nabla_{x} \rho\right)(y, z)=\left(\nabla_{y} \rho\right)(x, z) .
$$

Here, we remark that the latter equation is equivalent to requiring that the curvature tensor is harmonic, i.e. $\operatorname{div} R=0$.

In a similar way as in Theorem 3.3, we establish the truthfulness of the following

Theorem 3.4. The following characteristics of the manifold $(M, \varphi, \xi, \eta, g)$ are equivalent:

(i) The Ricci tensor of the manifold is of Codazzi type.

(ii) The manifold admits an almost Ricci-like soliton with vertical functions $(\lambda, \mu, v)$ satisfying the following equations

$$
\mathrm{d} \lambda=-\left\{f^{\prime}-\frac{f}{k}(\mu+v-f)\right\} \eta, \quad \mathrm{d} \mu=-\frac{f}{k} \mu \eta, \quad \mathrm{d} v=v^{\prime} \eta .
$$

(iii) The manifold is almost Einstein-like with vertical functions $(a, b, c)$ satisfying the following equations

$$
\mathrm{d} a=\frac{f}{k}(b+c) \eta, \quad \mathrm{d} b=-\frac{f}{k} b \eta, \quad \mathrm{d} c=c^{\prime} \eta .
$$

3.5. Ricci $\varphi$-symmetric manifolds. A manifold is called Ricci $\varphi$-symmetric if its non-vanishing Ricci operator $Q$, i.e. $\rho^{\sharp}$, satisfies the condition [9]

$$
\varphi^{2}\left(\nabla_{x} Q\right) y=0 .
$$

In [12], if the latter condition is satisfied for arbitrary vector fields on the manifold, it is called globally Ricci $\varphi$-symmetric, and when these vector fields are orthogonal to $\xi$, then the manifold is called locally Ricci $\varphi$-symmetric.

Using (20), we obtain the following expression

$$
\begin{aligned}
\varphi^{2}\left(\nabla_{x} Q\right) y= & -\mathrm{d}(\lambda+f)(x) \varphi^{2} y-\mathrm{d} \mu(x) \varphi^{2} y \\
& -\frac{f}{k} \mu \eta(y) \varphi^{2} x-\frac{f}{k}(\mu+v-f) \eta(y) \varphi^{2} x,
\end{aligned}
$$

It is not difficult to verify the validity of the following two statements.

Proposition 3.5. The manifold $(M, \varphi, \xi, \eta, g)$ is globally Ricci $\varphi$-symmetric if and only if it is Ricci symmetric. 
Theorem 3.6. The following characteristics of the manifold $(M, \varphi, \xi, \eta, g)$ are equivalent:

(i) The manifold is locally Ricci $\varphi$-symmetric.

(ii) The manifold admits an almost Ricci-like soliton with functions $(\lambda, \mu, v)$, where $\lambda$ and $\mu$ are vertical functions.

(iii) The manifold is almost Einstein-like with functions $(a, b, c)$, where $a$ and $b$ are vertical functions.

3.6. Manifolds with vanishing $Q \cdot R$. Let us consider the curvature condition $Q \cdot R=0$, which can be written in the form

(29) $R(x, y, z, Q w)-R(Q x, y, z, w)-R(x, Q y, z, w)-R(x, y, Q z, w)=0$.

Proposition 3.7. The manifold $(M, \varphi, \xi, \eta, g)$ satisfies the property $Q \cdot R=0$ if and only if it is Ricci flat.

Proof. Taking the trace of (29) for $x=e_{i}, w=e_{j}$ and due to the symmetry of $Q$, we obtain

$$
\rho(Q y, z)+\rho(y, Q z)=0 .
$$

After that we use (16) and get

$$
\left[(\lambda+f)^{2}-\mu^{2}\right] g(\varphi y, \varphi z)-2(\lambda+f) \mu g(y, \varphi z)-(\lambda+\mu+v)^{2} \eta(y) \eta(z)=0,
$$

which has a solution $(\lambda, \mu, v)=(-f, 0, f)$, i.e. the Ricci tensor vanishes according to (16).

3.7. Almost pseudo Ricci symmetric manifolds. In [7], the notion of almost pseudo Ricci symmetric manifolds is introduced by the following condition for its nonvanishing Ricci tensor

$$
\left(\nabla_{x} \rho\right)(y, z)=\{\alpha(x)+\beta(x)\} \rho(y, z)+\alpha(y) \rho(x, z)+\alpha(z) \rho(x, z),
$$

where $\alpha$ and $\beta$ are non-vanishing 1-forms.

Theorem 3.8. The manifold $(M, \varphi, \xi, \eta, g)$ is almost pseudo Ricci symmetric with 1-forms $\alpha$ and $\beta$ if and only if it is an almost Einstein-like manifold with functions $(a, b, c)$ relating to any of the following cases:

(i) $M$ is scalar flat (i.e. $\tau=0$ ) with a non-zero associated scalar curvature $\tau^{*}$ and

$$
(a, b, c)=\left(0,-\frac{\tau^{*}}{2 n}, \frac{\tau^{*}}{2 n}\right), \quad \alpha=-\frac{f}{k} \eta, \quad \beta=\left\{\frac{f}{k}+\frac{\mathrm{d} \tau^{*}(\xi)}{\tau^{*}}\right\} \eta .
$$

(ii) $M$ has non-zero $\tau$ and $\tau^{*}=-q \tau, q=$ const $\neq 0$, as well as

$$
(a, b, c)=\left(\frac{\tau}{2 n}, q \frac{\tau}{2 n},-(q+1) \frac{\tau}{2 n}\right), \quad \alpha=-\frac{f}{k} \eta, \quad \beta=\left\{\frac{f}{k}+\frac{\mathrm{d} \tau(\xi)}{\tau}\right\} \eta .
$$

(iii) $M$ is ${ }^{*}$-scalar flat (i.e. $\tau^{*}=0$ ) a non-zero scalar curvature $\tau$ and

$$
\begin{aligned}
& (a, b, c)=\left(\frac{\tau}{2 n}+\frac{1}{k^{2}}\left(k f^{\prime}+f^{2}\right), 0,-\frac{\tau}{2 n}-\frac{2 n+1}{k^{2}}\left(k f^{\prime}+f^{2}\right)\right), \\
& \alpha=-\frac{f}{k}\left\{1+2 n\left(k f^{\prime}+f^{2}\right)\right\} \eta, \\
& \beta=\left\{\frac{f}{k}\left[1+2 n\left(k f^{\prime}+f^{2}\right)\right]+\frac{\mathrm{d} a(\xi)}{a}\right\} \eta,
\end{aligned}
$$

where $k f^{\prime}+f^{2} \neq 0$ holds true, a is non-zero and it is given in (31). 
Proof. Combining (20) with (30) and (14), we obtain the following equality

$$
\begin{aligned}
& -\{\mathrm{d} a(x)-a[\alpha(x)+\beta(x)]\} g(\varphi y, \varphi z)+\{\mathrm{d} b(x)-b[\alpha(x)+\beta(x)]\} g(y, \varphi z) \\
& +\{\mathrm{d}(a+b+c)(x)-(a+b+c)[\alpha(x)+\beta(x)]\} \eta(y) \eta(z) \\
& -\frac{1}{k}\{f(b+c) \eta(y)-k a \alpha(y)\} g(\varphi x, \varphi z)-\frac{1}{k} b\{f \eta(y)+k \alpha(y)\} g(x, \varphi z) \\
& -\frac{1}{k}\{f(b+c) \eta(z)-k a \alpha(z)\} g(\varphi x, \varphi y)-\frac{1}{k} b\{f \eta(z)+k \alpha(z)\} g(x, \varphi y) \\
& -(a+b+c) \eta(x)\{\alpha(y) \eta(z)+\alpha(z) \eta(y)\}=0 .
\end{aligned}
$$

Substituting sequentially $\xi$ for $x, y, z$ and applying the result equalities in (32), we get the following condition

$$
\{f(b+c)-k a \alpha(\xi)\} g(\varphi x, \varphi y)+b\{f+k \alpha(\xi)\} g(x, \varphi y)-k(a+b+c) \eta(x) \alpha\left(\varphi^{2} y\right)=0 .
$$

which holds true for arbitrary $x, y$ and $z$ if and only if the following system of equalities satisfied:

$$
\begin{gathered}
f(b+c)-k a \alpha(\xi)=0, \quad b\{f+k \alpha(\xi)\}=0, \\
(a+b+c)\{\alpha-\alpha(\xi) \eta\}=0 .
\end{gathered}
$$

Solving the obtained system, we obtain the three solutions implying (i), (ii) and (iii), using (12) and (17).

Remark 3.9. For the first two cases in Theorem 3.8, the property $a+b+c=0$ is valid. Then, according to Remark 2.4, the vector field $\vartheta$ is not regular. In this case, due to (15), we have $k \mathrm{~d} f=-f^{2} \mathrm{~d} t$, where $t$ is a coordinate on $H^{\perp}$. Thus the solution is $f=k /\left(t-t_{0}\right)$, $t_{0}=$ const in the cases ( $i$ ) and (ii) of the latter theorem.

Theorem 3.10. The manifold $(M, \varphi, \xi, \eta, g)$ is almost pseudo Ricci symmetric with 1forms $\alpha$ and $\beta$ if and only if it admits an almost Ricci-like soliton with functions $(\lambda, \mu, v)$ relating to any of the following cases:

(i) $M$ is scalar flat (i.e. $\tau=0$ ) with a non-zero associated scalar curvature $\tau^{*}$ and

$$
(\lambda, \mu, v)=\left(-f, \frac{\tau^{*}}{2 n}, f-\frac{\tau^{*}}{2 n}\right), \quad \alpha=-\frac{f}{k} \eta, \quad \beta=\left\{\frac{f}{k}+\frac{\mathrm{d} \tau^{*}(\xi)}{\tau^{*}}\right\} \eta .
$$

(ii) $M$ has non-zero $\tau$ and $\tau^{*}=-q \tau, q=$ const $\neq 0$, as well as

$$
\begin{aligned}
& (\lambda, \mu, v)=\left(-f-\frac{\tau}{2 n},-q \frac{\tau}{2 n}, f+(q+1) \frac{\tau}{2 n}\right), \\
& \alpha=-\frac{f}{k} \eta, \quad \beta=\left\{\frac{f}{k}+\frac{\mathrm{d} \tau(\xi)}{\tau}\right\} \eta .
\end{aligned}
$$

(iii) $M$ is ${ }^{*}$-scalar flat (i.e. $\tau^{*}=0$ ) a non-zero scalar curvature $\tau$ and

$$
\begin{gathered}
(\lambda, \mu, v)=\left(-\frac{\tau}{2 n}-\frac{1}{k^{2}}\left(k f^{\prime}+f^{2}+k^{2} f\right), 0,\right. \\
\left.\frac{\tau}{2 n}+\frac{2 n+1}{k^{2}}\left(k f^{\prime}+f^{2}+k^{2} f\right)\right), \\
\alpha=-\frac{f}{k}\left\{1+2 n\left(k f^{\prime}+f^{2}\right)\right\} \eta, \\
\beta=\left\{\frac{f}{k}\left[1+2 n\left(k f^{\prime}+f^{2}\right)\right]+\frac{\mathrm{d}(\lambda+f)(\xi)}{\lambda+f}\right\} \eta,
\end{gathered}
$$

where $k f^{\prime}+f^{2} \neq 0$ and $\lambda+f \neq 0$ are valid, and $\lambda$ is given in (33).

Proof. The statement follows from Theorem 3.8 with equalities (14). 
3.8. Special weakly Ricci symmetric manifolds. A manifold is called special weakly Ricci symmetric if its non-vanishing Ricci tensor satisfies the following condition [26]

$$
\left(\nabla_{x} \rho\right)(y, z)=2 \alpha(x) \rho(y, z)+\alpha(y) \rho(x, z)+\alpha(z) \rho(x, z),
$$

where $\alpha$ is a non-vanishing 1 -form.

It is obvious that any special weakly Ricci symmetric manifold with 1-form $\alpha$ is almost pseudo Ricci symmetric with 1-forms $\alpha=\beta$. Thus Theorem 3.8 implies the following

Corollary 3.11. The manifold $(M, \varphi, \xi, \eta, g)$ is special weakly Ricci symmetric with 1form $\alpha$ if and only if it is an almost Einstein-like manifold with functions $(a, b, c)$ relating to any of the cases (i), (ii) and (iii) in Theorem 3.8 as well as the 1-form $\alpha$ is determined as follows:

(i) $\alpha=-\frac{f}{k} \eta=\frac{\mathrm{d} \tau^{*}(\xi)}{2 \tau^{*}} \eta$;

(ii) $\alpha=-\frac{f}{k} \eta=\frac{\mathrm{d} \tau(\xi)}{2 \tau} \eta$;

(iii) $\alpha=\frac{f c}{k a} \eta=\frac{\mathrm{d} a(\xi)}{2 a} \eta$, where $a$ and $c$ are given in (31).

As a consequence of (31) and (iii) in Corollary 3.11 we get the following differential condition for the scalar curvature and the conformal scalar

$$
k^{3} \tau^{\prime}+2 k^{2} f \tau=-2 n\left[k^{2} f^{\prime \prime}+4(n+1) k f f^{\prime}+2(2 n+1) f^{3}\right] .
$$

\section{EXAMPLE}

Let us consider the example from [20] of a manifold $\bar{M}:=\left(\mathbb{R}^{2 n+1}, \varphi, \xi, \eta, \bar{g}\right)$ belonging to $\mathcal{F}_{5} \backslash \mathcal{F}_{0}$, where the real space and the almost contact B-metric structure are defined as follows

$$
\begin{gathered}
\mathbb{R}^{2 n+1}=\left\{\left(x^{1}, \ldots, x^{n} ; x^{n+1}, \ldots, x^{2 n} ; t\right) \mid x^{i}, x^{n+i}, t \in \mathbb{R}, \quad i \in\{1,2, \ldots, n\}\right\}, \\
\varphi\left(\frac{\partial}{\partial x^{i}}\right)=\left(\frac{\partial}{\partial x^{n+i}}\right), \quad \varphi\left(\frac{\partial}{\partial x^{n+i}}\right)=-\left(\frac{\partial}{\partial x^{i}}\right), \quad \varphi\left(\frac{\partial}{\partial t}\right)=0, \\
\xi=\frac{\partial}{\partial t^{\prime}}, \quad \eta=\mathrm{d} t .
\end{gathered}
$$

Initially, a B-metric $g$ is introduced by the equality

$$
g(z, z)=-\delta_{i j}\left(z^{i} z^{j}-z^{n+i} z^{n+j}\right)+\left(z^{2 n+1}\right)^{2},
$$

where $z=z^{i} \frac{\partial}{\partial x^{i}}+z^{n+i} \frac{\partial}{\partial x^{n+i}}+z^{2 n+1} \frac{\partial}{\partial t}, i \in\{1,2, \ldots, n\}$ and $\delta_{i j}$ are the Kronecker's symbols. Then, the B-metric $\bar{g}$ is obtained by the following contact conformal transformation of $g$ determined by the functions $u$ and $v$ on $\mathbb{R}^{2 n+1}$ :

$$
\begin{aligned}
& \bar{g}=e^{2 u} \cos 2 v g+e^{2 u} \sin 2 v \tilde{g}+\left(1-e^{2 u} \cos 2 v-e^{2 u} \sin 2 v\right) \eta \otimes \eta, \\
& u=\frac{1}{2} \sum_{i=1}^{n}\left\{\ln \left[\left(x^{i}\right)^{2}+\left(x^{n+i}\right)^{2}\right]\right\}+\ell(t), \quad v=\sum_{i=1}^{n} \arctan \frac{x^{i}}{x^{n+i}},
\end{aligned}
$$

where $\ell(t)$ is an arbitrary twice differentiable function on $\mathbb{R}$ such that $\ell^{\prime} \neq 0$.

A torse-forming vertical vector field $\vartheta$ is considered, i.e. $\vartheta=k \xi$ and (10) is valid. Then, the conformal scalar $f$ of $\vartheta$ is determined by $f=k \ell^{\prime}$.

Next, it is shown that the obtained manifold is almost $\eta$-Einstein with functions

$$
a=-\frac{1}{k^{2}}\left[k f^{\prime}+2 n f^{2}\right], \quad b=0, \quad c=\frac{1-2 n}{k} f^{\prime}
$$


and scalar curvatures

$$
\tau=-\frac{2 n}{k^{2}}\left[2 k f^{\prime}+(2 n+1) f^{2}\right], \quad \tau^{*}=0
$$

Furthermore, in support of Corollary 2.5, case (i), the manifold $\bar{M}$ admits an almost Ricci-like soliton with potential $\vartheta$ and functions

$$
\lambda=\frac{1}{k^{2}}\left[k f^{\prime}+2 n f^{2}\right]-f, \quad \mu=0, \quad v=\frac{2 n-1}{k} f^{\prime}+f .
$$

We now determine the curvature properties studied in Section 3 of the resulting manifold $\bar{M}$ depending on the choice of the function $f$, bearing in mind that $f \neq 0$.

Using the characteristics of $\bar{M}$ given in (34), (35), (36), and the necessary and sufficient conditions given in Theorem 3.1, Theorem 3.2, Theorem 3.3, Theorem 3.4, Theorem 3.6, Proposition 3.7, Theorem 3.8 (iii), Theorem 3.10 (iii) and Corollary 3.11 (iii) in the respective cases, we obtain the following

Proposition 4.1. The constructed $(2 n+1)$-dimensional almost contact B-metric manifold $\bar{M}$ with a torse-forming vertical vector field $\vartheta=k \xi, k=$ const $\neq 0$, and a conformal scalar $f$ has the following properties:

(1) $\bar{M}$ is Ricci symmetric if and only if $f$ is constant. Then the resulting almost $\eta$-Ricci soliton is an $\eta$-Ricci soliton with a regular potential $\vartheta$ and constants

$$
\lambda=2 n \frac{f^{2}}{k^{2}}-f, \quad \mu=0, \quad v=f
$$

and $\bar{M}$ is Einstein with constant a and a negative constant scalar curvature $\tau$, where

$$
a=-2 n \frac{f^{2}}{k^{2}}, \quad \tau=-2 n(2 n+1) \frac{f^{2}}{k^{2}} .
$$

(2) $\bar{M}$ has vanishing $R(\xi,.) \cdot \rho$ if and only if $f$ is determined by

$$
f=\frac{k}{2 n\left(t-t_{0}\right)}, \quad t \neq t_{0}, \quad t_{0}=\text { const. }
$$

Then the scalar curvature is positive, $\tau=2 n(2 n-1) f^{2} / k^{2}$.

(3) $\bar{M}$ is Ricci cyclic parallel if and only if $f$ is constant. Then $\bar{M}$ is Einstein and $\tau$ is a negative constant, $\tau=-2 n(2 n+1) f^{2} / k^{2}$.

(4) $\bar{M}$ has a Ricci tensor of Codazzi type if and only if the function $f$ is determined by $k f^{\prime \prime}=-(2 n+1) f f^{\prime}$. A solution is the function

$f=\frac{2 k}{(2 n+1) t}, \quad t \neq 0$

and then $\bar{M}$ is scalar flat and almost $\eta$-Einstein with functions

$$
a=-\frac{2(2 n-1)}{(2 n+1)^{2} t^{2}}, \quad b=0, \quad c=\frac{2(2 n-1)}{(2 n+1) t^{2}} .
$$

(5) $\bar{M}$ is locally Ricci $\varphi$-symmetric for an arbitrary function $f$.

(6) $\bar{M}$ does not satisfy the property $Q \cdot R=0$ for an arbitrary function $f$.

(7) $\bar{M}$ is almost pseudo Ricci symmetric with 1-forms $\alpha$ and $\beta$ for an arbitrary function $f$ such that $k f^{\prime}+f^{2} \neq 0$. For example, let $f$ be determined by $f=-k / t$ for $t \neq 0$. Then, the manifold $\bar{M}$ has a negative scalar curvature $\tau=-2 n(2 n+3) / t^{2}$ and

$$
\begin{aligned}
& a=-\frac{2 n+1}{t^{2}}, \quad b=0, \quad c=-\frac{2 n-1}{t^{2}}, \\
& \lambda=\frac{k t+2 n+1}{t^{2}}, \quad \mu=0, \quad v=-\frac{k t-2 n+1}{t^{2}},
\end{aligned}
$$




$$
\alpha=\frac{t^{2}+4 n k^{2}}{t^{3}} \eta, \quad \beta=-\frac{3 t^{2}+4 n k^{2}}{t^{3}} \eta .
$$

(8) $\bar{M}$ is not special weakly Ricci symmetric for an arbitrary function $f$ with the condition $k f^{\prime}+f^{2} \neq 0$.

\section{REFERENCES}

[1] G. Ayar, M. Yıldırım, $\eta$-Ricci solitons on nearly Kenmotsu manifolds, Asian-European Journal of Mathematics 12 (2019) 2040002 (8 pages).

[2] C.S. Bagewadi, G. Ingalahalli, S. R. Ashoka, A study on Ricci solitons in Kenmotsu manifolds, International Mathematics Research Notices (2013) 412593 (6 pages).

[3] W. Batat, Curvature properties and Ricci solitons of Lorentzian pr-waves manifolds Journal of Geometry and Physics 75 (2014) 7-16.

[4] C. L. Bejan, S. Güler, Laplace, Einstein and related equations on D-general warping, Mediterranean Journal of Mathematics 16 (2019) 19.

[5] A. M. Blaga, $\eta$-Ricci solitons on para-Kenmotsu manifolds, Balkan Journal of Geometry and Its Applications 20 (2015) 1-13.

[6] A. M. Blaga, S. Y. Perktaş, Remarks on almost $\eta$-Ricci solitons in ( $\varepsilon$ )-para Sasakian manifolds, Communications Faculty of Sciences University of Ankara Series A1 Mathematics and Statistics 68 (2019) 1621-1628.

[7] M. C. Chaki, T. Kawaguchi, On almost pseudo Ricci symmetric manifolds, Tensor (N.S.) 68 (2007) 10-14.

[8] B. Chow, P. Lu, L. Ni, Hamilton's Ricci Flow, Graduate Studies in Mathematics 77, AMS Sci. Press, Providence, 2006.

[9] U.C. De, A. Sarkar, On $\varphi$-Ricci symmetric Sasakian manifolds, Proceedings of the Jangjeon Mathematical Society 11 (2008) 47-52.

[10] C. Gălin, M. Crasmareanu, From the Eisenhart problem to Ricci solitons in $f$-Kenmotsu manifolds, Bulletin of the Malaysian Mathematical Sciences Society 33 (2010) 361-368.

[11] G. Ganchev, V. Mihova, K. Gribachev, Almost contact manifolds with B-metric, Mathematica Balkanica (N.S.) 7 (1993) 261-276.

[12] S. Ghosh, U.C. De, On $\phi$-Ricci symmetric $(\kappa, \mu)$-contact metric manifolds, Acta Mathematica Universitatis Comenianae 86 (2017) 205-213.

[13] A. Gray, Einstein-like manifolds which are not Einstein, Geometriae Dedicata 7 (1978) 259-280.

[14] R. S. Hamilton, Three-manifolds with positive Ricci curvature, Journal of Differential Geometry 17 (1982) 255-306.

[15] S. Ivanov, M. Zlatanović, Non-symmetric Riemannian gravity and Sasaki-Einstein 5-manifolds, Classical and Quantum Gravity 37 (2020) 2, 025002.

[16] R. Ma, D. Pei, Some curvature properties on Lorentzian generalized Sasakian-space-forms, Advances in Mathematical Physics (2019) 5136758 (7 pages).

[17] H. Manev, D. Mekerov, Lie groups as 3-dimensional almost contact B-metric manifolds, Journal of Geometry 106 (2015) 229-242.

[18] M. Manev, Ricci-like solitons on almost contact B-metric manifolds, Journal of Geometry and Physics 154 (2020) 103734.

[19] M. Manev, Ricci-like solitons with vertical potential on Sasaki-like almost contact B-metric manifolds, Results in Mathematics 75 (2020) 136.

[20] M. Manev, Almost Ricci-like solitons with torse-forming vertical potential on Sasaki-like almost contact B-metric manifolds, arXiv:2006.07031.

[21] M. Manev, G. Nakova, Curvature properties of some three-dimensional almost contact B-metric manifolds, Plovdiv University Scientific Works - Mathematics 34 (2004) 51-60.

[22] A. Mihai, I. Mihai, Torse forming vector fields and exterior concurrent vector fields on Riemannian manifolds and applications, Journal of Geometry and Physics 73 (2013) 200-208.

[23] K. Onda, Lorentz Ricci solitons on 3-dimensional Lie groups. Geometriae Dedicata 147 (2010) 313-322.

[24] S. Pigola, M. Rigoli, M. Rimoldi, A. G. Setti, Ricci almost solitons, Annali della Scuola Normale Superiore di Pisa. Classe di Scienze. Serie V 10 (2011) 757-799.

[25] A. Singh, S. Kishor, Curvature properties of $\eta$-Ricci solitons on para-Kenmotsu manifolds, Kyungpook Mathematical Journal 59 (2019) 149-161.

[26] H. Singh, Q. Khan, On special weakly symmetric Riemannian manifolds, Publicationes Mathematicae Debrecen 58 (2001) 523-536.

[27] S. K. Yadav, S. K. Chaubey, D. L. Suthar, Certain results on almost Kenmotsu $(\kappa, \mu, v)$-spaces, Konuralp Journal of Mathematics 6 (2018) 128-133. 
CURVATURE PROPERTIES OF ALMOST RICCI-LIKE SOLITONS WITH TORSE-FORMING ...

[28] S.K. Yadav, S.K. Chaubey, D.L. Suthar, Some results of $\eta$-Ricci solitons on $(L C S)_{n}$-manifolds Surveys in Mathematics and its Applications 13 (2018) 237-250.

[29] S. K. Yadav, A. Kushwaha, D. Narain, Certain results for $\eta$-Ricci solitons and Yamabe solitons on quasi-Sasakian 3-manifolds, Cubo 21 (2019) 77-98.

[30] K. Yano, On torse forming direction in a Riemannian space, Proceedings of the Imperial Academy Tokyo 20 (1944) 340-345.

E-mail address: mmanev@uni-plovdiv.bg

(1) University of Plovdiv Paisi Hilendarski, Faculty of Mathematics and Informatics, Department of Algebra and Geometry, 24 Tzar Asen St., Plovdiv 4000, Bulgaria

(2) Medical University of Plovdiv, Faculty of Public Health, Department of Medical Informatics, Biostatistics and E-Learning, 15A Vasil Aprilov Blvd., Plovdiv 4002, Bulgaria 